\title{
Interaction between a verbal working memory network and the medial temporal lobe
}

\author{
Karl Magnus Petersson, ${ }^{\mathrm{a}, \mathrm{b}, \mathrm{c}, *}$ Jens Gisselgård, ${ }^{\mathrm{b}}$ Monica Gretzer, ${ }^{\mathrm{b}}$ and Martin Ingvar ${ }^{\mathrm{b}}$ \\ ${ }^{\mathrm{a}}$ F.C. Donders Centre for Cognitive Neuroimaging, Radboud University Nijmegen, The Netherlands \\ ${ }^{\mathrm{b}}$ Cognitive Neurophysiology Research Group, Department of Clinical Neuroscience, Karolinska Institutet, Sweden \\ ${ }^{\mathrm{C}} \mathrm{CSI}$, Centre for Intelligent Systems, Universidade do Algarve, Portugal
}

Received 2 August 2005; revised 4 July 2006; accepted 11 July 2006

Available online 29 September 2006

\begin{abstract}
The irrelevant speech effect illustrates that sounds that are irrelevant to a visually presented short-term memory task still interfere with neuronal function. In the present study we explore the functional and effective connectivity of such interference. The functional connectivity analysis suggested an interaction between the level of irrelevant speech and the correlation between in particular the left superior temporal region, associated with verbal working memory, and the left medial temporal lobe. Based on this psycho-physiological interaction, and to broaden the understanding of this result, we performed a network analysis, using a simple network model for verbal working memory, to analyze its interaction with the medial temporal lobe memory system. The results showed dissociations in terms of network interactions between frontal as well as parietal and temporal areas in relation to the medial temporal lobe. The results of the present study suggest that a transition from phonological loop processing towards an engagement of episodic processing might take place during the processing of interfering irrelevant sounds. We speculate that, in response to the irrelevant sounds, this reflects a dynamic shift in processing as suggested by a closer interaction between a verbal working memory system and the medial temporal lobe memory system.

(C) 2006 Elsevier Inc. All rights reserved.
\end{abstract}

\section{Introduction}

Cognitive functions are implemented in the network architecture of the brain. It is typically hypothesized that information is represented as distributed patterns of activity in these networks and that cognitive processing emerges as a consequence of the interaction between functionally specialized brain regions (e.g., Arbib, 2003; Ingvar and Petersson, 2000; Mesulam, 1998). A

\footnotetext{
* Corresponding author. F.C. Donders Centre for Cognitive Neuroimaging, PO Box 9101, NL-6500 HB Nijmegen, The Netherlands. Fax: +31 243610652. E-mail address: karl.magnus.petersson@ffcdonders.ru.nl

(K.M. Petersson).

Available online on ScienceDirect (www.sciencedirect.com).
}

central cognitive capacity is working memory which supports several higher cognitive functions, including language and reasoning (Baddeley, 1986, 2003). In the original Baddeley-Hitch model, working memory is composed of a central executive with two support systems, the phonological loop, storing verbal information, and the visuo-spatial sketch pad, storing visuo-spatial information. The functional-anatomical correlate of the phonological store is putatively the left inferior parietal region (IPC; Brodmann's area [BA] 39/40) together with parts of the superior temporal cortex (Becker et al., 1999; Paulesu et al., 1993), while the articulatory rehearsal process involves a left frontal circuit including Broca's region (BA 44) and the premotor cortex (BA 6, Smith and Jonides, 1998, 1999). Recently Baddeley (2000) added another component, the episodic buffer, a limited capacity system that provides temporary storage of information held in a multimodal code capable of binding information from the subsidiary systems and long-term memory into an episodic representational format (Fig. 1). The episodic buffer shares some characteristics with the concept of episodic memory encoding (Tulving, 1989) with respect to its principal mode of storing information in an episodic format as well as its integrative aspects, but differs in that it is assumed to be a capacity limited temporary store. Thus, the episodic buffer provides one interface between the original components of working memory and long-term memory. In emphasizing its short-term integrative role based on an episodic format, one may hypothesize that the episodic buffer is related to the prefrontal cortex (PFC) and the medial temporal lobe (MTL) as well as the interaction between these structures. The transient early role of the MTL system in long-term memory formation and sequence encoding in conjunction with the PFC makes these likely candidates (cf., e.g., Eichenbaum, 2000; Simons and Spiers, 2003).

Several recent functional neuroimaging studies have tentatively interpreted results in terms of the episodic buffer (e.g., Gruber, 2001; Gruber and von Cramon, 2003; Schon et al., 2004; Zhang et al., 2004). Gruber (2001) speculates that some of the prefrontal and parietal activations observed in an articulatory suppression paradigm might instantiate the episodic buffer, while Gruber and von Cramon (2003) suggest that the right middle frontal gyrus, the 


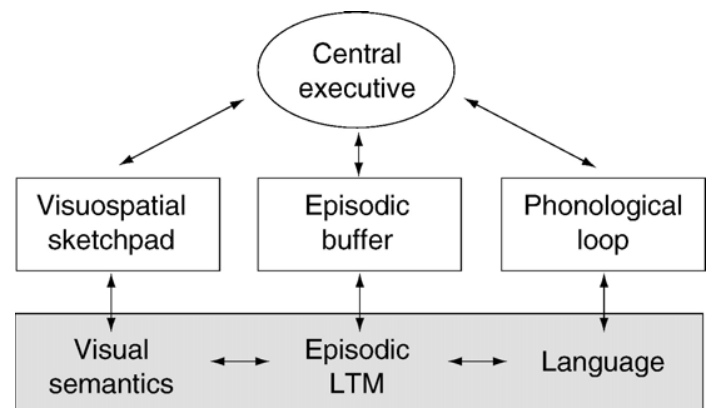

Fig. 1. The extended working memory model of Baddeley. The episodic buffer comprises a limited capacity system that provides temporary storage of information held in a multimodal code, capable of binding information from the subsidiary systems, and from long-term memory, into an episodic representational format. The episodic buffer is supposed to provide an interface to the other slave systems of working memory and to long-term memory, feeding information into and retrieving information from episodic long-term memory.

pre-SMA, bilaterally frontal opercular cortex and the cortex along the anterior and middle parts of the intraparietal sulcus are candidate regions for a hypothetical episodic buffer based on their observation that these regions showed memory-related activation independent of informational domain and memory strategy used. Zhang et al. (2004), following Baddeley's (2000) original suggestion, located the episodic buffer to the (right) prefrontal cortex based on the observation that this region was related to cross-modal integration or binding of information (see also Prabhakaran et al., 2000). Schon et al. (2004) on the other hand, suggest that persistent neural spiking in the perirhinal/entorhinal cortex during the delay period in a delayed match-to-sample task might constitute a cellular basis of the episodic buffer. A somewhat related perspective on the role of the medial temporal lobe as well as the prefrontal and posterior cortical regions in working memory, albeit not necessarily tied to a concept of the episodic buffer, have recently been outlined by Cabeza et al. (2002), Ranganath and Blumenfeld (2005) and Ranganath (2006).

In the extraction of information from the working memory stores a key function for the episodic buffer is to integrate between the different subcomponents. One behavioral effect that seems well suited to model such interplay is the irrelevant speech effect (Baddeley, 1997). This effect refers to a reduction in the immediate serial recall of lists of presented items, usually digits or letters, when irrelevant auditory material is presented together with the items to be memorized. The irrelevant speech effect occurs despite the fact that the sounds are irrelevant to the serial recall task at hand and that subjects are explicitly instructed to ignore the speech (Jones, 1993). The effect also occurs regardless of whether the items to be memorized are presented visually (Salame and Baddeley, 1982) or auditorily (Hanley and Broadbent, 1987), whether the irrelevant speech occurs at presentation or during the retention interval (Miles et al., 1991), or whether the irrelevant sounds comprise meaningful or meaningless information (Colle and Welsh, 1976). In a recent study (Gisselgård et al., 2003) we reported that the irrelevant speech effect was associated with a relative decrease of activation in the left superior temporal gyrus (STG) and a similar, but weaker, activity decrease in the right homotopic region (Fig. 2a). We suggested that the activity decrease observed in the superior temporal region might reflect a suppression of early phonological processing. In addition, and related to the irrelevant speech effect, relative decreases of activation were found in the inferior/middle frontal cortices and the left inferior parietal cortex, that were interpreted as a suppression of activity in several neural components related to the phonological loop (Paulesu et al., 1993; Smith and Jonides, 1999). This suppression might be realized as a modulation by some other brain region, for example, the anterior cingulate cortex (ACC) or the PFC. Moreover, since the subjects were able to fully compensate behaviorally for the auditory interference, additional brain regions are likely to be recruited for compensatory processing. The episodic buffer provides for one possible compensation mechanism. In this context it is of interest to note that there is evidence suggesting that when the working memory load exceeds some level, a processing shift occurs from phonological coding to other forms of coding, for example episodic or semantic (Baddeley, 1966a,b; Hanley and Bakopoulou, 2003; Larsen and Baddeley, 2003; Salame and Baddeley, 1986).

In the first analysis of the present study, we investigated the functional connectivity of the superior temporal region where the irrelevant speech effect was expressed (Gisselgård et al., 2003) in an attempt to understand its role in verbal working memory by reanalyzing the data from our previous study of the irrelevant speech effect (Gisselgård et al., 2003). This revealed a negative correlation between the left STG and the left MTL. We interpreted this finding tentatively as reflecting an interaction between verbal working memory and the MTL system, induced by a transition in mnemonic processing during irrelevant speech. In order to further understand this finding within a more comprehensive framework, we analyzed the observed covariance structure using structural equations modeling in the second analysis of the present study (Bollen, 1989; McIntosh and Gonzalez-Lima, 1994). We adapted a previously described network model (Petersson et al., 2000) which includes simple models for the phonological loop, the central executive, attentional modulation as well as articulatory motor output. The network approach attempts to characterize the functional interactions between regions in terms of effective connections in a specific functional-anatomical model. Given our speculation about the instantiation of the episodic buffer outlined above, the focus of these investigations was the functional interactions between prefrontal regions (associated with manipulation and active maintenance of representations in working memory), the inferior parietal region (associated with the phonological store component), and the medial temporal lobe (representing episodic/semantic recoding). We hypothesized that these regions would interact more intensely at high levels of irrelevant speech, interpreted as reflecting an increased utilization of the episodic buffer.

\section{Materials and methods}

\section{Participants}

Eleven right-handed healthy male subjects with a similar shortterm memory span (10-40\% errors in the single pseudoword condition) were recruited to the foregoing PET study (for further details see Gisselgård et al., 2003) from a behavioral study sample of 30 .

\section{Stimuli and task procedures}

In brief the experimental paradigm consisted of a $2 \times 3$ factorial design (memory load $\times$ irrelevant speech). The memory load had 
a

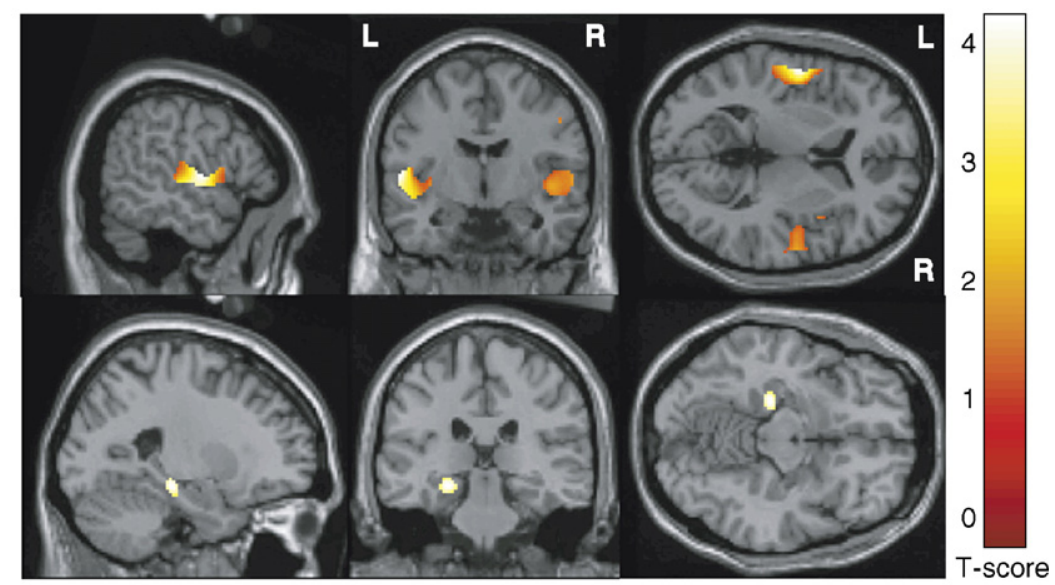

C

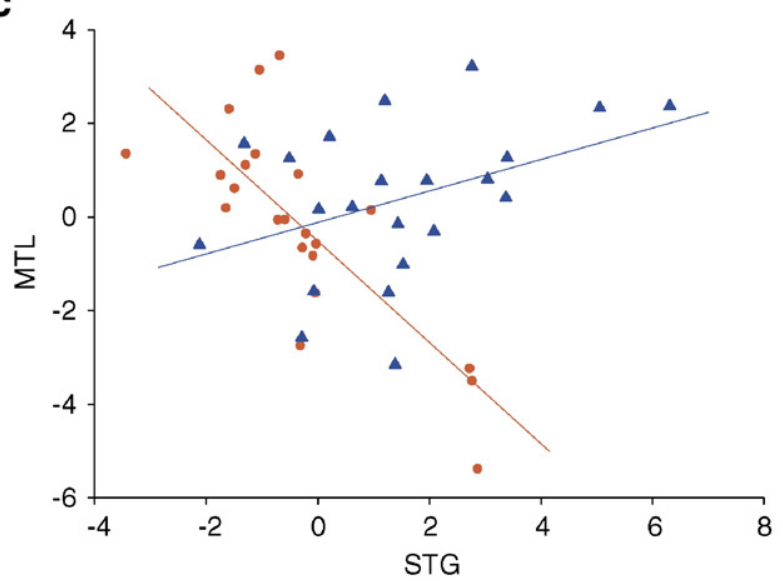

Fig. 2. Interaction between superior temporal cortex and medial temporal lobe. (a) Activity in the interaction contrast between the level of irrelevant speech (single vs. multiple items) and working memory load (serial recall vs. counting). The rCBF changes are displayed on an MR-overlay, showing the deactivation of the bilateral superior temporal cortices. For display purposes, pixels exceeding a threshold level of significance of $P<0.05$ uncorrected for multiple comparisons are displayed on sagittal, coronal and transverse projections of the brain. (b) The activation of the left hippocampus as revealed by functional connectivity analysis when using the signal from a seed voxel in the left superior temporal cortex. (c) Scatter plots of the interaction (repeated measures ANOVA: $P=0.04$ ) in functional connectivity between the two different irrelevant speech conditions during serial recall; single CV-items and multiple different CV-items. The taskrelated signal change from the voxel in the left superior temporal gyrus (STG, stereotactic coordinates $[-54,-6,6])$ showing maximal signal change in the irrelevant speech interaction contrast is plotted against the voxel the medial temporal region that displayed a significant negative correlation with the STG in functional connectivity analysis (stereotactic coordinates $[-24,-28,-12]$ ).

two levels: immediate serial recall of different digit sequences and immediate serial repetition of the standard digit sequence from one to six. There were three different levels of irrelevant speech: quiet, single CV-syllables, and multiple different CV-syllables. In this study we only analyzed the data from the single and multiple CV-syllables conditions, since these two irrelevant speech conditions are closely matched in terms of sensory input and motor output and thus not confounded by factors which we are not interested in. In addition, there was no performance difference between these conditions. The subjects engaged in six encodingrecall cycles. After the word "Ready" was displayed on the computer screen a list was presented. The digits were recalled immediately in serial order after encoding. The subjects practiced all aspects of the experimental paradigm for approximately $20 \mathrm{~min}$ in the PET scanner before the experiment started. Each condition was repeated twice with unique lists for each scan. Each digit was shown $0.5 \mathrm{~s}$ and the inter-digit interval was $0.5 \mathrm{~s}$. The time for recall was $4 \mathrm{~s}$. The irrelevant speech (i.e., the CV-syllables) was auditorily presented with headphones at a level of $65 \mathrm{~dB}$. The irrelevant speech in the single item condition consisted of repetitions of the identical CV-pseudoword 'da', while the CVpseudowords 'ne', 'li', 'to', 'vu', 'py', 'bå', 'nö' were used in the multiple items condition and presented in a randomized order. For further details concerning the PET procedures see Gisselgård et al. (2003).

\section{Functional connectivity analysis}

For the functional connectivity analysis, the activity of the voxel $([x, y, z]=[-54,-6,6])$ showing maximal signal change in the irrelevant speech contrast (Gisselgård et al., 2003) was extracted and entered into a correlation analysis within the general linear model framework of SPM99. The extracted signal change of the voxel was corrected with respect to condition-mean and separated on conditions as additional regressors to the condition regressors in a linear model, thus allowing for the investigation of correlation-by-condition interactions and also revealing regions of 
positive or negative correlation in a given condition as well as their comparisons.

\section{Functional-anatomical network model}

The network model was based on the Baddeley-Hitch model of verbal working memory (Baddeley, 1992) that includes a phonological (online) store and an articulatory rehearsal process. Functional neuroimaging data (Paulesu et al., 1993) have suggested that the phonological loop network is subserved by the superior temporal (BA 22), the inferior parietal (BA 39/40), and the inferior frontal (BA 44/6) regions. In particular, it has been hypothesized that aspects of the phonological store are related to the inferior parietal region while aspects of the articulatory control process are related to inferior frontal region (cf., e.g., Baddeley, 2000). Functional neuroimaging studies have also indicated that aspects of the central executive are localized to the left middle-inferior prefrontal cortex (BA 45/46, e.g., D'Esposito et al., 1995; Petrides, 1995; Petrides et al., 1993, 1995). For a comprehensive empirical review see Cabeza and Nyberg (2000). It should be emphasized that the relation between the cognitive components of the Baddeley-Hitch model and their suggested neural substrata in terms of a detailed information processing account is not well understood at present.

The network model for verbal working memory has to explain a sufficient part of the observed covariance, in both the single and the multiple CV-syllable condition, and be both theoretically and empirically plausible. Data from the regions included in the model (Table 1, Fig. 3) were sampled from representative voxels of the different regions and the voxels can be viewed as weighted spherical regions of interest as the imaging data were spatially filtered $(\mathrm{FWHM}=14 \mathrm{~mm})$. In the following, $\rightarrow$ denotes a unidirectional connection while $\leftrightarrow$ denotes a recurrent connection, that is, a feed-forward and feed-back connection that are allowed to differ in connection strength.

We included the left STG (BA 22/42) connected to the left posterior part of inferior frontal gyrus (IFG, BA 44; STG $\rightarrow$ IFC) and a simple articulatory motor output circuit: the left primary motor cortex for articulation $(\mathrm{M}, \mathrm{BA} 4$; IFC $\rightarrow \mathrm{M}$ ) and since the right lateral cerebellar region has been related to certain aspects of language processing (e.g., Cabeza and Nyberg, 2000), this region was included in the articulatory output circuit $(\mathrm{Cdx}$; IFC $\rightarrow \mathrm{Cdx})$. The model was extended to include the phonological loop as described above: the left inferior parietal cortex (IPC, BA 39/40) and its connections to the left STG and the left IFC (STG $\leftrightarrow$ IPC $\leftrightarrow$ IFC). This core was extended to include the anterior cingulate cortex

Table 1

The different regions included in the network model

\begin{tabular}{llll}
\hline Region & & Brodmann's area & {$[X, Y, Z]$} \\
\hline Superior temporal gyrus & STG & BA 22/42 & {$[-54,-6,6]$} \\
Inferior parietal cortex & IPC & BA 39/40 & {$[-44,-48,42]$} \\
Anterior cingulate cortex & ACC & BA 32 & {$[-4,10,42]$} \\
Prefrontal cortex & PFC & BA 45/46 & {$[-50,26,20]$} \\
Inferior frontal cortex & IFC & BA 44 & {$[-52,6,20]$} \\
Primary motor cortex & M & BA 4 & {$[-52,-6,40]$} \\
Medial temporal lobe & MTL & BA 35 & {$[-24,-28,-12]$} \\
Right cerebellum & Cdx & & {$[36,-60,-22]$} \\
\hline
\end{tabular}

Data from the regions were sampled from representative voxels comparable to weighted spherical regions of interests since the PET data were spatially smoothed with an isotropic Gaussian kernel of FWHM=14 mm.

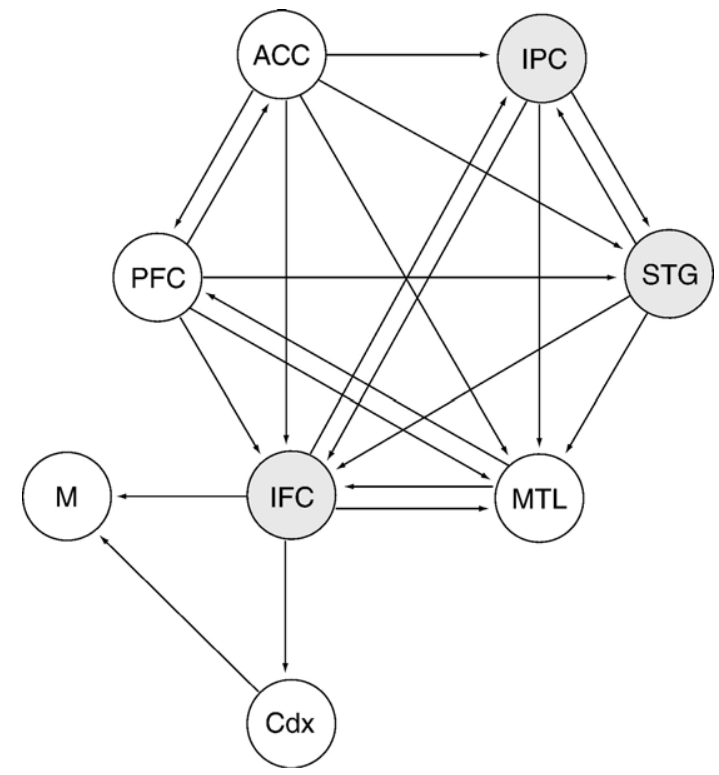

Fig. 3. The network topology of our verbal working memory model. The phonological loop (grey circles) consists of two sub-systems: the phonological store (IPC, inferior parietal cortex BA 39/40, and STG, superior temporal gyrus BA 22/42) and the articulatory control process (IFC, inferior frontal cortex BA 44). Attentional control and central executive functions are hypothesized to be related to the ACC (anterior cingulated cortex BA 32) and the PFC (dorsolateral prefrontal cortex BA 45/46), respectively. The MTL (medial temporal lobe) memory system is related to episodic processing and declarative memory. A simple articulatory output sub-network encompasses M (primary articulatory motor cortex BA 4) and the right lateral cerebellum $(\mathrm{Cdx})$.

(ACC) hypothesized to be related to attention, error detection and response competition/selection (Carter et al., 1998) and the left middle-inferior dorsolateral prefrontal region (PFC, BA 45/46) suggested to subserve aspects of the central executive. The ACC and PFC are often activated in concert (e.g., Petersson et al., 1999a) and both regions likely subserve different executive aspects of prefrontal processing, reflected here in the recurrent connection ACC $\leftrightarrow$ PFC. Further connections, related to the ACC, included ACC $\rightarrow$ STG/ $\mathrm{IPC} / \mathrm{IFC} / \mathrm{MTL}$ (short hand for $\mathrm{ACC} \rightarrow \mathrm{STG}, \mathrm{ACC} \rightarrow \mathrm{IPC}$, etc.), as a simple model for attentional modulation. Based on the suggestion of an interaction between the prefrontal cortex and the MTL (e.g., Nyberg et al., 2003; Petersson et al., 1999a), we included the recurrent connections PFC $\leftrightarrow$ MTL and IFC $\leftrightarrow$ MTL. Finally, given the observed correlation between the STG and MTL, we included the connection STG $\rightarrow$ MTL, and, as a simple model for the influence of the posterior parietal cortex on the MTL, we also included IPC $\rightarrow$ MTL.

\section{Network analysis}

SEM models are parameterized by the connection strengths (path coefficients), the residuals, and the variances/covariances among and between these parameters and use a linear system of equations to describe the interrelations of activities between the regions of the functional-anatomical model (Bollen, 1989; McIntosh and Gonzalez-Lima, 1994). To maintain estimability, certain constraints in the model selection procedure are implemented in terms of constraints on parameters that can be specified. Following Petersson et al. 
(2000), in the present study the residuals were fixed to $66 \%$ of the corresponding observed variance and the covariances between residuals were set to zero (cf., McIntosh and Gonzalez-Lima, 1994). The connection strengths were estimated with an iterative maximum likelihood optimization process using the LISREL software (version 8.52, Scientific Software, Inc., Jöreskog and Sörbom, 1989). For each model, this process also yields a goodness-of-fit estimate (the maximum likelihood distance between implied and observed covariance structure). In the context of multivariate normally distributed variables, the goodness-of-fit index is $\chi^{2}$ distributed with $(q / 2)(q+1)-p$ degrees of freedom, where $p$ is the number of free parameters and $q$ is the number of observed variables (Bollen, 1989). The goodness-of-fit index can be translated into a $P$-value that indicates how well the model explains or represents the observed covariance structure. This implies that a large goodness-offit $P$-value ( $>0.05$, cf., Gonzalez-Lima and McIntosh, 1994) is desirable and indicates that a sufficient part of the observed covariance structure can be explained by the model. Differences between conditions were tested with stacked models analysis. In a stacked analysis the connections strengths are estimated under the constraint that the corresponding connections are equal across groups or states. If the underlying assumptions are valid, then the $P$ value reflects the goodness-of-fit of the null hypothesis that the same set of network connections can explain the observed covariance structures across conditions, that is, if the cognitive processing is qualitatively and quantitatively similar, as reflected in the pattern of network interactions, in the different conditions. Stacked analyses may also be performed on sub-networks by letting the complementary part of the network be free while the connection strengths of the sub-network of interest are constrained to be the same.

There is a trade-off between the degree of elaboration of the model and estimability properties. In the present study, we used a mixed approach, being theory driven in specifying the regions and the outline of the network topology based on a previously investigated model, and fine tuning the precise connection configuration in order to achieve comparable as well as sufficiently high goodness-of-fit values in both conditions. Note that this was done before any hypothesis testing was undertaken and thus blind to any differential effects between conditions. The model selection was based on the following principles: connections were excluded if they were small in terms of the absolute value of the connection strength or were similar in both conditions and did not carry any significant explanatory value in terms of goodness-of-fit. In particular this was the case if these connections related to parts of the network not central to the focus of investigation, allowing us to prioritize central parts of the network and model these in a more elaborate manner. For example, PFC $\leftrightarrow$ IPC, which may be natural on theoretical grounds as well as previous experimental evidence, were initially included but did not entail any significant explanatory value (low connection strengths in both conditions, replicating the experience of Petersson et al., 2000). Furthermore, during the model selection stage we also investigated the robustness properties of such models in general, indicating relatively stable results when different subsets of what was judged to be less important connections were excluded from the model (Gretzer, 2003).

\section{Assumptions and limitations of the network approach to effective connectivity}

The network approach described here is a covariance-based approach. The basic assumption of various covariance approaches is that the intrinsic variability in the neural response, during a cognitive state or a particular task, will emulate the relevant functional interactions or effective connectivity. It is assumed that these interactions and the underlying dynamic functional architecture (Petersson, 2005) are reflected in the observed covariance structure (Petersson et al., 2000). Two major ways of estimating the covariances within a cognitive state have been described: across subjects (Horwitz et al., 1995), over time/repetitions within subject (Buchel and Friston, 1997), or both (Buchel et al., 1999). In this context, it should be noted that the sources of within-state interregional covariances are beyond experimental control (Petersson et al., 1999b). Several sources of interregional covariances have been proposed (Horwitz et al., 1992) and the actual sources of the observed covariances are largely unknown. However, some of the proposed sources might potentially give rise to spurious correlations that are necessarily confounded with the correlations arising because of the effective connectivity or the functional architecture. Obvious potential confounds in the study of interregional covariances are global effects (for a review see e.g., Petersson et al., 1999b). In addition, when the covariances are estimated over subjects, it is necessary to assume that the subjects implement a sufficiently similar functional organization. In this case, the covariance structure may reflect an average common functional architecture. In PET studies the number of intra-subject observations is limited (restricted by radiation exposure) and the data are typically pooled over subjects in order to increase sensitivity. With fMRI data, it is possible to study functional and effective connectivity in single subjects (e.g., Buchel and Friston, 1997). A distinct advantage with single-subject studies is that these provide information on the generalizability of the results if these are subjected to statistical analysis in for example a meta-analytic approach.

The results of structural equations modeling (SEM) are potentially difficult to interpret for several reasons. For example, there is no guarantee that the connections modeled reflect direct effective connections; it is possible that they are mediated through areas or connections not included in the model. Similarly, observed changes in the weights between states or groups may reflect common input from regions not modeled. This touches on the problem of model selection. In the case of SEM, model selection may be performed in a data-driven manner, guided by goodness-of-fit values, modification indices or using a hierarchical model building approach when subsets of weights are estimated recursively (McIntosh and Gonzalez-Lima, 1994). The data-driven approach is vulnerable to over-fitting since sample specific characteristics may be modeled (e.g., noise and outliers). Investigating over-fitted models can limit the generalizability of results. Alternatively, model selection may be theory driven, running the risk of investigating incomplete models. Moreover, unless reasonable goodness-of-fit can be achieved with a given model in all conditions or groups investigated, the results of a stacked models comparison can be difficult to interpret. For example, using an under-parameterized model to test differences between conditions or groups in a stacked analysis may yield results due to an ill-fitting model in one of the conditions or groups. The effect of using under-parameterized models has been investigated to some extent in a simple network model (McIntosh and Gonzalez-Lima, 1994). These simulations indicate that the results from analyzing moderately reduced models are fairly stable and that the modification indices can provide indications of missing connections or regions in the model chosen. It should also be noted that the maximum likelihood 
estimation of the SEM approach assumes that a global optimum has been reached. The standard implementation in LISREL uses instrumental variables and a two-stage least square approach in combination with the Davidon-Fletcher-Power algorithm and line search to find a (local) optimum of the objective function, which in practice often is close to the global optimum (cf., e.g., Bollen, 1989; Jöreskog and Sörbom, 1996). In general, the objective function to be optimized is a complicated nonlinear function of the model parameters and often an explicit global optimum solution is not known (Bollen, 1989). In addition, it is possible that the objective function has several local optima. In such a case, there is no guarantee that the global optimum will be reached with deterministic gradient descent algorithms or nonexhaustive search procedures. Alternatively, stochastic search algorithms like for example a simulated annealing approach to optimization can be used (Kirkpatrick and Sorkin, 1995; Kirkpatrick et al., 1983), but these are typically computationally intensive. These and additional issues are discussed further in Petersson et al. $(1999 b, c)$.

\section{Results}

As a background to the results of the functional connectivity and network analyses we note that there was no difference between the two conditions (single and multiple CV-syllable conditions) at a behavioral level (the probability of recall in the single CV-syllable condition: $91 \%$ ( $\pm 3 \mathrm{SE})$; and in the multiple $\mathrm{CV}$-syllable condition: $92 \%$ ( $\pm 3 \mathrm{SE}$ ); $P>0.50$, n.s.; for further details see Gisselgård et al., 2003).

\section{Functional connectivity}

In the functional connectivity study of the left STG, the most prominent finding was a correlation between the left STG (BA 22/ $42,[x, y, z]=[-54,-6,6])$ and the left MTL $([-24,-28,-12]$, Fig. b) that was modulated by the level of irrelevant speech; the correlation was marginally positive in the single CV-syllable immediate serial recall condition $\left(\beta=0.34, R^{2}=0.16, F_{1,20}=3.84\right.$, $P=0.06$, uncorrected), while this was negative in the multiple $\mathrm{CV}$ syllable condition $\left(\beta=-1.1, R^{2}=0.60, F_{1,20}=30, P<0.001\right.$, uncorrected). Scatter plots of the task-related signal change in the left STG and the left MTL is plotted in Fig. 2c. The psychophysiological interaction (i.e., condition $\times$ correlation interaction) between the STG and the MTL suggested a difference $(P<0.001$, uncorrected) in the functional connectivity as a function of the level of irrelevant speech (i.e., single and multiple CV-syllable condition) during immediate serial recall digit span task. However, since this SPM analysis used the within-subject residual this is akin to a fixed effects analysis, we also analyzed the data with a repeated measures ANOVA (random effects analysis), and importantly, the psycho-physiological interaction was significant $\left(F_{1,10}=5.29, P=0.04\right)$.

\section{Network interactions}

The goodness-of-fit for our model corresponded to $P>0.20$ in both the single and multiple item condition, indicating that the network model (Fig. 3) was able to represent the observed covariance structure sufficiently well in both conditions. The global stacked models analysis, testing for differences between conditions, indicated that the single and multiple item condition differed significantly $(P=0.008)$ in terms of the pattern of network interactions as captured by the connection strengths (Fig. 4). We then proceeded to test different functional sub-networks for differences between conditions. No sub-network that we investigated differed significantly between conditions (cognitive sub-networks $P \geq 0.26$, articulatory output sub-network $P \geq 0.15$ ) except for the connections related to the MTL $(P=0.006)$. More

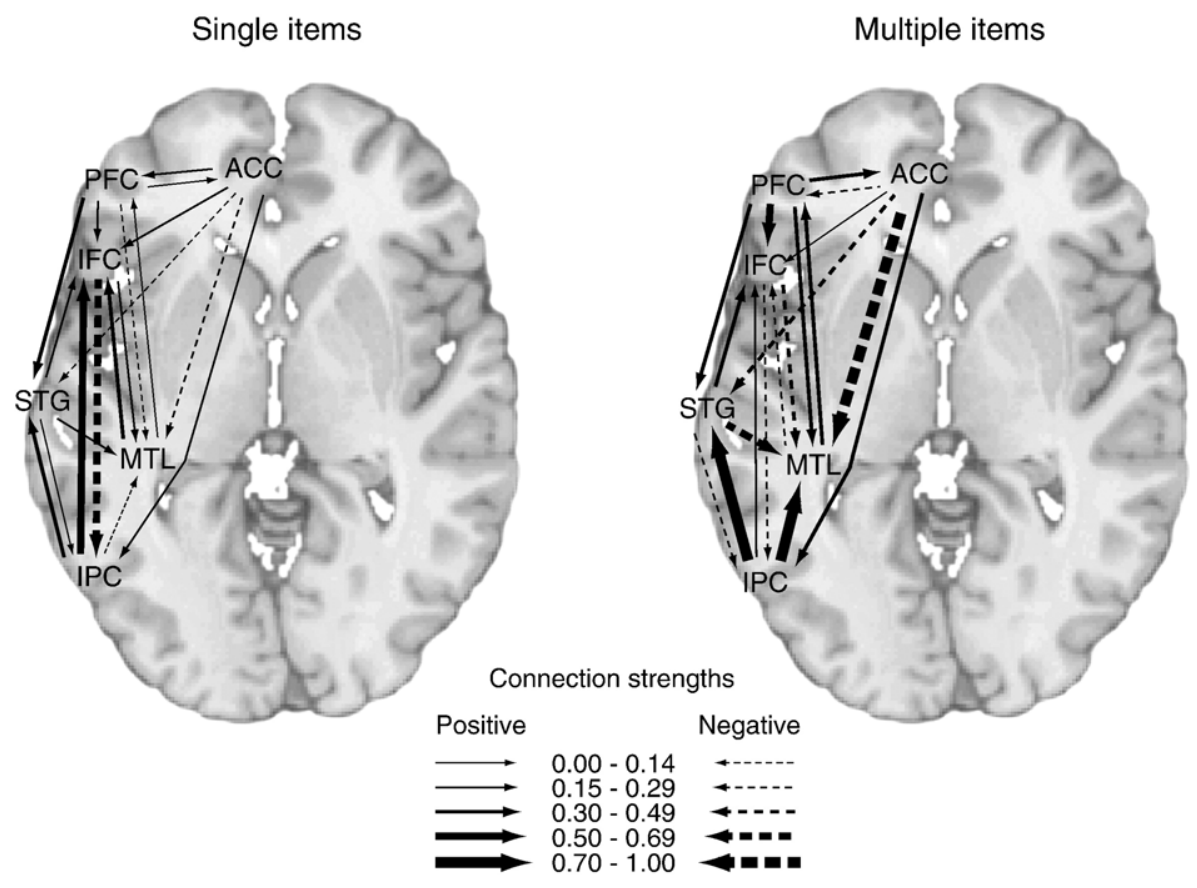

Fig. 4. Connection strengths in the single and multiple item conditions. The goodness-of-fit for the network model corresponded to $P>0.20$ in both conditions. This indicates that the model is able to explain, or represent, the observed covariance structure reasonably well in both conditions. 


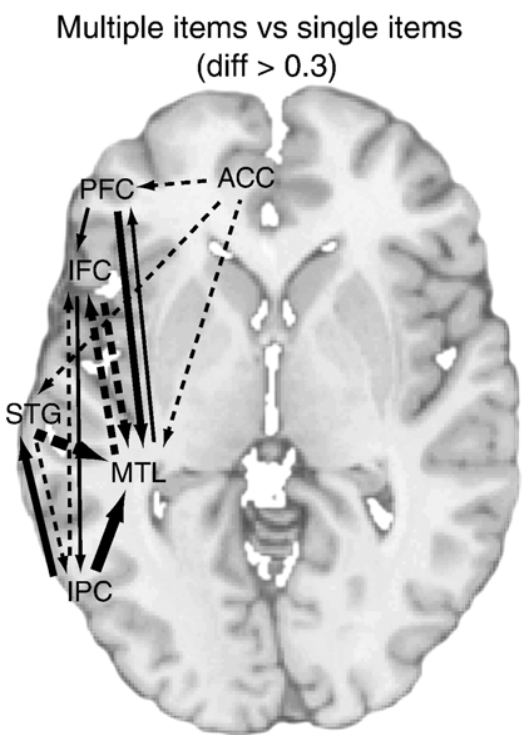

Connection strengths

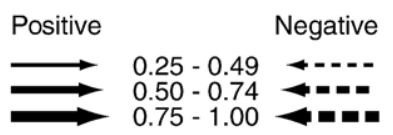

Fig. 5. The relative difference in connection strengths between the multiple and single item conditions $(\mathrm{M}-\mathrm{S})$. No meaningful functional sub-network differed significantly between conditions except for the connections related to the MTL $(P=0.006)$. Only differences exceeding 0.25 (standardized values) in absolute value are shown.

specifically, the interactions between the MTL and the phonological loop (IPC $\rightarrow$ MTL, IFC $\leftrightarrow$ MTL, $P=0.005$; STG $\rightarrow$ MTL, PFC $\leftrightarrow$ MTL, $P=0.005)$. We further focused our attention to the interaction between the prefrontal regions and the MTL, which showed marginally significant differences between conditions (PFC/IFC $\leftrightarrow$ MTL $P=0.059)$, as well as the interaction between the posterior regions and MTL (STG/IPC $\rightarrow \mathrm{MTL}$, IPC $\leftrightarrow$ STG, $P=0.077$; STG/ $\mathrm{IPC} \rightarrow$ MTL, $P=0.057)$.

In summary, the results suggest a dissociation in terms of network interactions, as characterized in terms of connection strength changes, between PFC (multiple $>$ single item) and IFC (single $>$ multiple item) as well as STG (single $>$ multiple item) and IPC (multiple $>$ single item) in relation to the MTL. The results of the functional connectivity analyses are thus consistent with this pattern of observations. We discuss this set of results further in the discussion section, in particular with reference to Fig. 5.

\section{Discussion}

The initial motivation for this study was to further characterize the role of the STG with respect to the irrelevant speech effect. To this end, we investigated the functional connectivity of this region with the rest of the brain. The most prominent finding was a correlation between the left STG and the left MTL that was sensitive to the single- vs. multiple CV-syllable manipulation during serial recall of digits. The subsequent network analysis indicated that this finding was part of a more general phenomenon suggesting more intense interaction between the verbal working memory network and the MTL as expressed in changes of the connection strengths related to the level of irrelevant speech. This was most clearly expressed as increases in the connections: PFC $\leftrightarrow$ MTL, IPC $\leftrightarrow$ MTL; and decreases in the following connections: $\mathrm{STG} \rightarrow \mathrm{MTL}, \mathrm{IFC} \rightarrow \mathrm{MTL}$, and ACC $\rightarrow$ MTL (Fig. 5).

The initial STG-MTL results were intriguing since the MTL has typically been associated with episodic long-term memory and MTL lesions do not typically produce severe deficits related to short-term memory (e.g., Eichenbaum and Cohen, 2001; however, see Ranganath and Blumenfeld, 2005). One might argue that, since the multiple items condition contains greater variability in the irrelevant speech input and perhaps to some degree relatively more novel information compared to the single item condition, the present result simply reflects an episodic encoding effect. We suggest that this is not the case, because we observed a positive correlation between the left STG and the left MTL in the single item condition, while the reverse was the case for the multiple items condition (Fig. 2c). Instead we suggest that the observed difference may reflect a change in the interaction between these regions, depending on the level of irrelevant speech, and related to a processing shift between the two conditions. The results of the network analysis support this interpretation, because, given the changes in functional STG-MTL connectivity as a function of irrelevant speech level, one would predict that the other related systems should show parallel changes in interaction pattern. In this context, it is of interest to note that there is a discussion in the literature concerning whether the MTL plays a role in working memory and whether the MTL is necessary for memory over brief delays (e.g., Cabeza et al., 2002; Ranganath, 2006; Ranganath and Blumenfeld, 2005; Ranganath and D'Esposito, 2001; Zarahn et al., 2005). Zarahn et al. (2005) review some of the evidence that suggests that the MTL plays a role in memory over brief delays and conclude that it appears that the human MTL is necessary not only for memory over brief delays for complex/novel stimuli, but also for familiar stimuli under some circumstances. In particular, it is suggested that in the presence of a perceived possibility of distraction, memory for familiar stimuli over brief delays might be dependent on the integrity of the MTL. Zarahn et al. (2005) conclude that the functional neuroimaging results (Ranganath and D'Esposito, 2001; Zarahn et al., 2005) are consistent with their synthesis from the lesion literature which supports the idea that the MTL has a role in memory over brief delays in humans. In addition, Cabeza et al. (2002) reported overlapping activations in the MTL related to episodic retrieval and verbal working memory. On their re-examination of the literature, Cabeza et al. (2002) argued, that there is evidence linking MTL regions with working memory and similar conclusions were reached by Ranganath and Blumenfeld (2005; see also Ranganath, 2006); for example, in both human (Buffalo et al., 1998; Holdstock et al., 1995; Owen et al., 1995) and nonhuman primates (Murray and Mishkin, 1986; Zola et al., 2000), MTL lesions have been found to impair performance in trial unique working memory tasks with retention intervals no longer than a few seconds (for a comprehensive review see Eichenbaum and Cohen, 2001). There is also electrophysiological (Cahusac et al., 1993; Suzuki et al., 1997), autoradiographical (Davachi and Goldman-Rakic, 2001), and functional neuroimaging (Curtis et al., 2000; Elliott and Dolan, 1999; Piekema et al., in press; Ranganath and D'Esposito, 2001; Schon et al., 2004) evidence that the MTL is active during working memory tasks. Moreover, several computational models have investigated the interaction between short-term working and long-term memory or the interaction between neocortical regions and the MTL in similar 
terms (Jensen and Lisman, 1996; O’Reilly et al., 1999). Furthermore, multiple memory systems operating at different relevant time-scales can be instantiated in sets of dynamical variables with different characteristic time-constants in the same network (Sandberg et al., 2002).

The finding of sustained MTL activity during delay periods in humans is consistent with evidence for sustained unit activity in the MTL (parahippocampal and entorhinal cortex) during delayed matching tasks in nonhuman primates and rats (e.g., Suzuki et al., 1997; Young et al., 1997). Recent intracellular recording data suggest a potential mechanism for mediating this sustained unit activity for novel stimuli in parahippocampal structures (Egorov et al., 2002; Klink and Alonso, 1997). The active maintenance of information in parahippocampal structures is essential in a number of computational MTL models (Hasselmo et al., 2002; Jensen and Lisman, 1996). Schon et al. (2004) suggest that this mechanism might constitute a cellular basis of an episodic buffer.

We have previously suggested that the interaction between working memory and long-term memory also involves prefrontal and posterior parietal regions (Petersson et al., 1997, 1999a). Consistently with this, overlap in neocortical activation patterns between verbal working memory and episodic memory, including commonalities related to the prefrontal and posterior parietal regions, have recently been reported (Nyberg et al., 2002, 2003). The inferior parietal cortex putatively represents the online phonological store, while the STG is the prime receiver of the irrelevant speech. In the multiple item, high interference condition, we suggest that the observed processing shift, as reflected in the increased connection strength IPC $\rightarrow$ MTL, might be related to the generation of a real-time representation in an 'episodic' format. Naturally, this does not exclude incidental episodic long-term memory formation, since, as already noted, multiple memory systems can be instantiated in the same network using dynamical variables operating at different characteristic time-scales. However, this is neither a necessary or sufficient processing consequence for successful working memory performance in the present case.

As the IPC engages the MTL, this region also increases its connection strength to the STG, and at the same time, the STG decreases its interactive influence over the MTL and the IPC. This suggests that the IPC may attempt to override the irrelevant input represented in the STG. In parallel, the intensity of the interaction between the left PFC, reflecting aspects of central executive processing, and the left MTL increases. We suggest this might represent an attempt to organize the relevant information into an episodic representational format in interaction with the MTL. In addition, on the output side, the PFC increase its interactive control over the IFC, perhaps representing a reinterpretation of the newly created episodic format into a phonological output format suitable for the organization of an articulatory response, as required by the IFC and reflected in the relative increase in the connection strength PFC $\rightarrow$ IFC (Fig. 5). In this context, we propose additional suggestive interpretations of the increase related to IFC $\rightarrow$ IPC and the decrease related to IPC $\rightarrow$ IFC. The decrease in interaction strength between the IFC and the MTL as well as the decreased connection strength IPC $\rightarrow$ IFC, may be interpreted as an attempt to avoid an intermixing of the phonological output representation with the episodic representation in the MTL, and, the irrelevant phonological input (represented in the IPC) and the articulatory output format (represented in the IFC), when the relevant representation is created in the MTL (from which the articulatory output is subsequently derived via the PFC). Thus, we suggest that the decrease in IPC $\rightarrow$ IFC reflects a decrease of interactive information flow from the IPC to the IFC, the commonly held view of phonological loop operation, and instead the relevant information is increasingly shuttled via the MTL and the PFC to the IFC, reflecting the suggested processing shift. At the same time, the increase in IFC $\rightarrow$ IPC might reflect an attempt to recurrently close the phonological loop, via articulatory rehearsal, in order to support the relevant information content during the relatively greater interference from the irrelevant speech.

Finally, we will attempt to integrate the suggested interpretations above with the newly introduced episodic buffer, the fourth component of the extended Baddeley-Hitch working memory (Baddeley, 2000). The episodic buffer comprises a limited capacity system that provides temporary storage of information held in a multimodal code, which is capable of binding information from the subsidiary systems, and from long-term memory, into an episodic representational format (Fig. 1). Similar concepts, including long-term working memory and working-with-memory, have been suggested by Ericsson and Kintsch (1995) and Moscovitch (1994), respectively. Cabeza et al. (2002) suggested that, at the neuronal level, rehearsal processes may involve the reactivation of the working memory representations transiently stored in the neocortex, and that accessing these representations might engage the MTL. In addition, it has been suggested that the MTL may serve as a convergence zone, rapidly storing arbitrary associations or conjunctions of information, binding different distributed neocortical representations active at the time of encoding, storing an index of or pointer to neocortical representations, so-called chunking (Wickelgren, 1979). One possibility is that, under some circumstances, such pointers may be used to access working memory traces transiently stored in neocortex and that similar MTL mechanisms may be engaged to serve several functions during online processing. For example, Cabeza et al. (2002), following Miller (1991), suggested that working memory might use MTL indexing mechanisms to access short-term memory representations (see also Ranganath, 2006), whereas episodic memory may use MTL indexing to encode and retrieve long-term memory information. Since, the episodic buffer provides an interface between the support systems of working memory and long-term memory, an interesting possibility in the context of this study is that the episodic buffer is instantiated as an interaction between the PFC and the MTL, possibly also including the posterior parietal cortex. Given the close relation between episodic memory and the MTL, an observation of interest in relation to immediate serial recall is that when task difficulty increases there is a tendency for subjects to abandon phonological coding in favor of other strategies (Salame and Baddeley, 1986). Such a strategy switch would provide a potential remedy against the detrimental influence of interfering or disturbing stimuli. Thus our observations might reflect a strategy shift, from pure working memory processing, to an engagement of capacities related to episodic processing and by implication the MTL, reflecting integrative aspects of on-going information processing. However, it should be pointed out that a similar perspective on the role of the medial temporal lobe as well as the prefrontal and posterior cortical regions in working memory, which is not dependent on the concept of an episodic buffer, can be argued and have recently been outlined by Cabeza et al. (2002) and Ranganath (2006). Under all circumstances, these perspectives remain controversial 
since patients with MTL lesions do not typically show dramatic short-term memory deficits. However, it should be noted that short-term memory investigations in MTL lesion patients typically have used simple short-term memory tasks and a potential explanation may be that these are not sensitive enough to detect subtle short-term memory deficits. Such deficits may be more pronounced if an additional distracting task or disturbing input is delivered and this hypothesis can be tested directly on patients with MTL lesions.

\section{Conclusion}

We suggest that an interactive integrative perspective on the relation between neocortical working memory and the medial temporal lobe memory system is fruitful. The results of the present study supports this notion and suggest a seamless transaction between these two conceptually defined cognitive systems, in the present case, switching from a relatively pure processing of phonological representations to engage in processing of episodic representations. This entails that different memory systems interact dynamically according to specific task demands. We suggest that our results reflect a dynamic shift in processing as suggested by a closer interaction between a verbal working memory system and the medial temporal lobe system, a possible instantiation of the episodic buffer.

\section{Acknowledgments}

This work was supported by grants from the Swedish Medical Research Council [8276], the Karolinska Institutet, the Swedish Bank Tercentenary Foundation and the Knut and Alice Wallenberg Foundation.

\section{References}

Arbib, M.A. (Ed.), 2003. The Handbook of Brain Theory and Neural Networks, 2 ed. MIT Press, Cambridge, MA.

Baddeley, A., 1966a. The influence of acoustic and semantic similarity on long-term memory for word sequences. Q. J. Exp. Psychol. 18, 302-309.

Baddeley, A., 1966b. Short-term memory for word sequences as a function of acoustic, semantic and formal similarity. Q.J. Exp. Psychol. 18, $362-365$.

Baddeley, A., 1986. Working Memory. Oxford Univ. Press, Oxford.

Baddeley, A., 1992. Working memory. Science 255, 556-559.

Baddeley, A., 1997. Human Memory: Theory and Practice, Revised ed. Earlbaum.

Baddeley, A., 2000. The episodic buffer: a new component of working memory? Trends Cogn. Sci. 4, 417-423.

Baddeley, A., 2003. Working memory and language: an overview. J. Commun. Disord. 36, 189-208.

Becker, J.T., MacAndrew, D.K., Fiez, J.A., 1999. A comment on the functional localization of the phonological storage subsystem of working memory. Brain Cogn. 41, 27-38.

Bollen, K.A., 1989. Structural Equations with Latent Variables. Wiley, New York.

Buchel, C., Friston, K.J., 1997. Modulation of connectivity in visual pathways by attention: cortical interactions evaluated with structural equation modelling and fMRI. Cereb. Cortex 7, 768-778.

Buchel, C., Coull, J.T., Friston, K.J., 1999. The predictive value of changes in effective connectivity for human learning. Science 283, 1538-1541.

Buffalo, E.A., Reber, P.J., Squire, L.R., 1998. The human perirhinal cortex and recognition memory. Hippocampus 8, 330-339.
Cabeza, R., Nyberg, L., 2000. Imaging cognition II: an empirical review of 275 PET and fMRI studies. J. Cogn. Neurosci. 12, 1-47.

Cabeza, R., Dolcos, F., Graham, R., Nyberg, L., 2002. Similarities and differences in the neural correlates of episodic memory retrieval and working memory. NeuroImage 16, 317-330.

Cahusac, P.B.M., Miyashita, Y., Rolls, E., 1993. Response of hippocampal formation neurons in the monkey related to delayed spatial response and object-place memory tasks. Behav. Brain Res. 33, 229-240.

Carter, C.S., Braver, T.S., Barch, D.M., Botvinick, M.M., Noll, D., Cohen, J.D., 1998. Anterior cingulate cortex, error detection, and the online monitoring of performance. Science 280, 747-749.

Colle, H.A., Welsh, A., 1976. Acoustic masking in primary memory. J. Verbal Learn. Verbal Behav. 15, 17-32.

Curtis, C.E., Zald, D.H., Lee, J.T., Pardo, J.V., 2000. Object and spatial alternation tasks with minimal delays activate the right anterior hippocampus proper in humans. NeuroReport 11, 2203-2207.

Davachi, L., Goldman-Rakic, P.S., 2001. Primate rhinal cortex participates in both visual recognition and working memory tasks: functional mapping with 2-DG. J. Neurophysiol. 85, 2590-2601.

D’Esposito, M., Detre, J.A., Alsop, D.C., Shin, R.K., Atlas, S., Grossman, M., 1995. The neural basis of the central executive system of working memory. Nature 378, 279-281.

Egorov, A.V., Hamam, B.N., Fransen, E., Hasselmo, M.E., Alonso, A.A., 2002. Graded persistent activity in entorhinal cortex neurons. Nature 420, 173-178.

Eichenbaum, H., 2000. A cortical-hippocampal system for declarative memory. Nat. Rev., Neurosci. 1, 41-50.

Eichenbaum, H., Cohen, N.J., 2001. From Conditioning to Conscious Recollection: Memory Systems of the Brain. Oxford Univ. Press, Oxford, UK.

Elliott, R., Dolan, R.J., 1999. Differential neural responses during performance of matching and nonmatching to sample tasks at two delay intervals. J. Neurosci. 19, 5066-5073.

Ericsson, K.A., Kintsch, W., 1995. Long-term working memory. Psychol. Rev. 102, 211-245.

Gisselgård, J., Petersson, K.M., Baddeley, A., Ingvar, M., 2003. The irrelevant speech effect: a PET study. Neuropsychologia 41, 1899-1911.

Gonzalez-Lima, F., McIntosh, A.R., 1994. Neural network interactions related to auditory learning analyzed with structural equation modeling. Hum. Brain Mapp. 2, 23-44.

Gretzer, M., 2003. Structural Equation Modeling in Neuroimaging: Application and Evaluation of the Method. Unpublished Masters Thesis, Royal Institute of Technology, TRITA-NA-E03082, Stockholm, Sweden.

Gruber, O., 2001. Effects of domain-specific interference on brain activation associated with verbal working memory task performance. Cereb. Cortex 11, 1047-1055.

Gruber, O., von Cramon, D.Y., 2003. The functional neuroanatomy of human working memory revisited Evidence from 3-T fMRI studies using classical domain-specific interference tasks. NeuroImage 19, 797-809.

Hanley, J.R., Bakopoulou, E., 2003. Irrelevant speech, articulatory suppression, and phonological similarity: a test of the phonological loop model and the feature model.[comment]. Psychon. Bull. Rev. 10, 435-444.

Hanley, J.R., Broadbent, C., 1987. The effects of unattended speech on serial recall following auditory presentation. Br. J. Psychol. 78, 287-297.

Hasselmo, M.E., Cannon, R.C., Koene, R.A., 2002. A simulation of parahippocampal and hippocampal structures guiding spatial navigation of a virtual rat in a virtual environment: a functional framework for theta theory. In: Wouterlood, F.G. (Ed.), The Parahippocampal Region: Organization and Role in Cognitive Functions. Oxford Univ. Press, Oxford, UK, pp. 139-161.

Holdstock, J.S., Shaw, C., Aggleton, J.P., 1995. The performance of amnesic subjects on test of delayed matching-to-sample and delayed matchingto-position. Neuropsychologia 33, 1538-1596. 
Horwitz, B., Soncrant, J.V., Haxby, J.V., 1992. Covariance analysis of functional interactions in the brain using metabolic and blood flow data. In: Scheich, H. (Ed.), Advances in Metabolic Mapping Techniques for Brain Imaging of Behavioral and Learning Functions. Kluwer Academic Publishing, Dordrecht, The Netherlands, pp. 189-217.

Horwitz, B., McIntosh, A.R., Haxby, J.V., Grady, C.L., 1995. Network analysis of brain cognitive function using metabolic and blood flow data. Behav. Brain Res. 66, 187-193.

Ingvar, M., Petersson, K.M., 2000. Functional maps-cortical networks. In: Toga, A.W., Mazziotta, J.C. (Eds.), Brain Mapping: The Systems. Academic Press, San Diego, CA, pp. 111-140.

Jensen, O., Lisman, J.E., 1996. Novel lists of 7+-2 known items can be reliably stored in an oscillatory short-term memory network: interaction with long-term memory. Learn. Mem. 3, 257-263.

Jones, C., 1993. Objects, streams, and threads of auditory attention. In: Weiskrantz, L. (Ed.), Attention: Selection, awareness, and control. Oxford Univ. Press, Oxford, England, pp. 87-104.

Jöreskog, K., Sörbom, D., 1989. LISREL 7 User's Reference Guide. Scientific Software, Mooresville, IN.

Jöreskog, K., Sörbom, D., 1996. LISREL 8 User's Reference Guide. Scientific Software International, Chicago, IL.

Kirkpatrick, S., Sorkin, G.B., 1995. Simulated annealing. In: Arbib, M.A. (Ed.), The Handbook of Brain Theory and Neural Networks. MIT Press, Cambridge, MA, pp. 876-879.

Kirkpatrick, S., Gelatt, C.D., Vecchi, M.P., 1983. Optimization by simulated annealing. Science 220, 671-680.

Klink, R., Alonso, A.A., 1997. Muscarinic modulation of the oscillatory and repetitive firing properties of entorhinal cortex layer II neurons. J. Neurophysiol. 77, 1813-1828

Larsen, J.D., Baddeley, A., 2003. Disruption of verbal STM by irrelevant speech, articulatory suppression, and manual tapping: do they have a common source?[see comment]. Q. J. Exp. Psychol., A $56,1249-1268$.

McIntosh, A.R., Gonzalez-Lima, F., 1994. Structural equation modeling and its application to network analysis in functional brain imaging. Hum. Brain Mapp. 2, 2-22.

Mesulam, M.M., 1998. From sensation to cognition. Brain 121, 1013-1052.

Miles, C., Jones, D.M., Madden, C.A., 1991. Locus of the irrelevant speech effect in short-term memory. J. Exper. Psychol., Learn., Mem., Cogn. 17, $578-584$.

Miller, R., 1991. Cortico-Hippocampal Interplay and the Representation of Contexts in the Brain. Springer-Verlag, Berlin.

Moscovitch, M., 1994. Memory and working with memory: evaluation of a component process model and comparisons with other models. In: Tulving, E. (Ed.), Memory Systems. MIT Press, Cambridge, MA, pp. 269-310.

Murray, A.C., Mishkin, M., 1986. Visual recognition in monkeys following rhinal cortical ablations combined with either amygdalectomy or hippocampectomy. J. Neurosci. 6, 1991-2003.

Nyberg, L., Forkstam, C., Petersson, K.M., Cabeza, R., Ingvar, I., 2002. Brain imaging of human memory systems: between-systems similarities and within-system differences. Cogn. Brain Res. 13, 281-292.

Nyberg, L., Marklund, P., Persson, J., Cabeza, R., Forkstam, C., Petersson, K.M., Ingvar, I., 2003. Common prefrontal activations during working memory, episodic memory, and semantic memory. Neuropsychologia 41, 371-377.

O'Reilly, R.C., Braver, T.S., Cohen, J.D., 1999. A biologically based computational model of working memory. In: Shah, P. (Ed.), Models of Working Memory: Mechanisms of Active Maintenance and Executive Control. Cambridge University Press, Cambridge, UK.

Owen, A.M., Sahakian, B.J., Semple, J., Polkey, C.E., Robins, T.W., 1995. Visuo-spatial short-term recognition memory and learning after temporal lobe excision, frontal lobe excision or amygdalo-hippocampectomy in man. Neuropsychologia 33, 1-24.

Paulesu, E., Frith, C.D., Frackowiak, R.S., 1993. The neural correlates of the verbal component of working memory. Nature 362, 342-345.
Petersson, K.M., 2005. Learning and Memory in the Human Brain Karolinska Univ. Press, Stockholm, Sweden.

Petersson, K.M., Elfgren, C., Ingvar, M., 1997. A dynamic role of the medial temporal lobe during retrieval of declarative memory in man. NeuroImage 6, 1-11.

Petersson, K.M., Elfgren, C., Ingvar, M., 1999a. Dynamic changes in the functional anatomy of the human brain during recall of abstract designs related to practice. Neuropsychologia 37, 567-587.

Petersson, K.M., Nichols, T.E., Poline, J.-B., Holmes, A.P., 1999b. Statistical limitations in functional neuroimaging I: non-inferential methods and statistical models. Philos. Trans. R. Soc. Lond., B 354 $1239-1260$.

Petersson, K.M., Nichols, T.E., Poline, J.-B., Holmes, A.P., 1999c. Statistical limitations in functional neuroimaging II: signal detection and statistical inference. Phil. Trans. R. Soc. Lond., B 354, 1261-1282.

Petersson, K.M., Reis, A., Askelöf, S., Castro-Caldas, A., Ingvar, M., 2000. Language processing modulated by literacy: a network analysis of verbal repetition in literate and illiterate subjects. J. Cogn. Neurosci. 12 (3), 364-382.

Petrides, M., 1995. Functional organization of the human frontal cortex for mnemonic processing. Evidence from neuroimaging studies. Ann. N. Y. Acad. Sci. 769, 85-96.

Petrides, M., Alivisatos, B., Meyer, E., Evans, A.C., 1993. Functional activation of the human frontal cortex during the performance of verbal working memory tasks. Proc. Natl. Acad. Sci. U. S. A. 90, 878-882.

Petrides, M., Alivisatos, B., Evans, A.C., 1995. Functional activation of the human ventrolateral frontal cortex during mnemonic retrieval of verbal information. Proc. Natl. Acad. Sci. U. S. A. 92, 5803-5807.

Piekema, C., Kessels, R.P.C., Mars, R.B., Petersson, K.M., Fernández, G. in press. The right human hippocampus participates in active maintenance of object-location associations. NeuroImage (doi:10.1016/j. neuroimage.2006.06.035).

Prabhakaran, V., Narayanan, K., Zhao, Z., Gabrieli, J.D., 2000. Integration of diverse information in working memory within the frontal lobe. Nat. Neurosci. 3, 85-90.

Ranganath, C., 2006. Working memory for visual objects: complementary roles of inferior temporal, medial temporal, and prefrontal cortex. Neuroscience 139, 277-289.

Ranganath, C., Blumenfeld, R.S., 2005. Doubts about double dissociations between short- and long-term memory. Trends Cogn. Sci. 9, 374-380.

Ranganath, C., D'Esposito, M., 2001. Medial temporal lobe activity associated with active maintenance of novel information. Neuron 31 , $865-873$

Salame, P., Baddeley, A., 1982. Disruption of short-term memory by irrelevant speech: implications for the structure of working memory J. Verbal Learn. Verbal Behav. 21, 150-164.

Salame, P., Baddeley, A.D., 1986. Phonological factors in STM: similarity and the unattended speech effect. Bull. Psychon. Soc. 24, 263-265.

Sandberg, A., Lansner, A., Petersson, K.M., Ekeberg, Ö., 2002. Bayesian attractor networks with incremental learning. Netw.: Comput. Neural Syst. 13, 179-194.

Schon, K., Hasselmo, M.E., LoPresti, M.L., Tricarico, M.D., Stern, C.E., 2004. Persistence of parahippocampal representation in the absence of stimulus input enhances long-term encoding: a functional magnetic resonance imaging study of subsequent memory after a delayed matchto-sample task. J. Neurosci. 24, 11088-11097.

Simons, J.S., Spiers, H.J., 2003. Prefrontal and medial temporal lobe interactions in long-term memory. Nat. Rev., Neurosci. 4, 637-648.

Smith, E.E., Jonides, J., 1998. Neuroimaging analyses of human working memory. Proc. Nat. Acad. Sci. U. S. A. 95, 12061-12068.

Smith, E.E., Jonides, J., 1999. Storage and executive processes in the frontal lobes. Science 283, 1657-1661.

Suzuki, W.A., Miller, E.K., Desimone, R., 1997. Object and place memory in macaque entorhinal cortex. J. Neurophysiol. 78, 1062-1081.

Tulving, E., 1989. Memory: performance, knowledge and experience. Eur. J. Cogn. Psychol. 1, 3-26. 
Wickelgren, W.A., 1979. Chunking and consolidation: a theoretical synthesis of semantic networks, configuring, S-R versus cognitive learning, normal forgetting, the amnesic syndrome, and the hippocampal arousal system. Psychol. Rev. 86, 44-60.

Young, B.J., Otto, T., Fox, G.D., Eichenbaum, H., 1997. Memory representation within the parahippocampal region. J. Neurosci. 17, 5183-5195.

Zarahn, E., Rakitin, B., Abela, D., Flynn, J., Stern, Y., 2005. Positive evidence against human hippocampal involvement in working memory maintenance of familiar stimuli. Cereb. Cortex 15, 303-316.

Zhang, D., Zhang, X., Sun, X., Li, Z., Wang, Z., He, S., Hu, X., 2004. Crossmodal temporal order memory for auditory digits and visual locations: an fMRI study. Hum. Brain Mapp. 22, 280-289.

Zola, S.M., Squire, L.R., Teng, E., Stefanacci, L., Buffalo, E.A., Clark, R.E., 2000. Impaired recognition memory in monkeys after damage to the hippocamplal region. J. Neurosci. 20, 451-463. 